\title{
Electrical twitch obtaining intramuscular stimulation (ETOIMS) for myofascial pain syndrome in a football player
}

\author{
J Chu, I Takehara, T-C Li, I Schwartz
}

Br J Sports Med 2004;38:e25 (http://www.bjsportmed.com/cgi/content/full/38/5/e25). doi: 10.1136/bjsm.2003.010306

\begin{abstract}
Background: Flare up of acute lower back pain associated with myofascial pain syndrome (MPS) may require various forms of treatment including activity restriction and bracing. Electrical twitch obtaining intramuscular stimulation (ETOIMS) is a promising new treatment. It involves the use of a strong monopolar electromyographic needle electrode for electrical stimulation of deep motor end plate zones in multiple muscles in order to elicit twitches.

Case report: An elite American football player with MPS symptoms failed to respond to standard treatments. He then received ETOIMS which completely alleviated the pain. After establishing pain control, the athlete continued with a further series of treatments to control symptoms of muscle tightness. Conclusions: ETOIMS has a promising role in pain alleviation, increasing and maintaining range of motion, and in providing satisfactory athletic performance during long term follow up.
\end{abstract}

$\mathrm{T}$ he only well established risk factor that predisposes athletes to recurrent injury is previous muscle strain. Past lower back pain increases the risk of a later recurrence of back pain and lower limb muscle strain injuries. ${ }^{2}$ Our case presentation highlights the use of a novel technique termed "electrical twitch obtaining intramuscular stimulation" (ETOIMS) ${ }^{4}$ in an elite American football player to alleviate myofascial pain and improve athletic function.

\section{CASE REPORT}

A 21 year old male college football wide-receiver complained of progressive bilateral (left predominate) chronic lower back pain and tight hip muscles for three years before seeking medical attention at our institution in order to decrease his pain and increase his athletic performance. Symptoms started after a bilateral fracture of L5 pars interarticularis associated with repetitive $136 \mathrm{~kg}$ squat lifts and $295 \mathrm{~kg}$ leg presses. Although the fracture healed, he continued to have chronic lower back and hip pain. Despite activity restriction, bracing, physical therapy, and acupuncture, pain was $8 / 10$ on a visual analogue scale (VAS).

On initial examination, the patient had no localising neurological findings but spinal extension and lateral bending were mildly limited, although flexion was full. He was unable to perform a full squat. T7-S1 paraspinal muscles and the lower limb muscles, especially on the left, had tender myofascial trigger points. Electromyography revealed no spontaneous activity, but the left L2 and bilateral L5 myotomes showed an increased percentage of polyphasic motor unit action potentials. The patient underwent ETOIMS with manual insertion of a monopolar needle electrode into multiple motor points at $2 \mathrm{~Hz}$ for two seconds, using unipolar negative wave of $20 \mathrm{~mA}$ and $0.5 \mathrm{~ms}$ pulse duration, supplied by the EMG machine. The muscles treated were primarily bilateral lower paraspinals, gluteus maximus, tensor fascia latae, adductor magnus, and rectus femoris.

Outcome measures included serial VAS and the ability to flex, abduct, and externally rotate (FABERE) the hips before each treatment. FABERE measurement was from the lateral end of the ipsilateral knee crease to the bed surface. The subject received consecutive weekly treatments, but for analysis we arbitrarily divided the sessions into "pain present" and "pain-free" series-that is, 10 and 13 consecutive sessions, respectively. In the "pain present" series, left FABERE measurements correlated positively with VAS level $(r=0.7, \mathrm{p}=0.009)$ and negatively with number of treatments $(r=-0.58, \mathrm{p}=0.05)$. No further correlations occurred between "pain-free" series for left FABERE or for both series for the right FABERE. However, treatment resulted in an overall decrease in maximum FABERE and pain. During 2.5 years of follow up, the patient returned for two more treatments, one session each, at five month intervals. FABERE measurements showed more limitation but remained improved from pretreatment values (fig 1). After college, he continues as a professional wide-receiver, returning for treatments for continued pain relief and to improve his agility, speed, and endurance.

\section{DISCUSSION}

Tender myofascial trigger points in MPS which produce referred pain and a local twitch response on snapping palpation are identical to motor end plate zones. ${ }^{5}$ Treatment of tender myofascial trigger points includes injections such as local anaesthetic, steroids, and botulinum toxin, but injections have a limited role when multiple trigger points in many muscles need chronic repetitive treatments. Through limiting the role of oral or injected drugs for pain relief and functional restoration, ETOIMS has a decided advantage in sports related recurrent MPS. It is contraindicated for patients with local or systemic infections, autoimmune deficiencies, and bleeding disorders.

Limitations of acupuncture with or without electricity, percutaneous electrical nerve stimulation, and intramuscular stimulation $^{6}$ include inability to stimulate deep motor end plate zones. These procedures employ acupuncture needles with a diameter of $0.25 \mathrm{~mm}$ and lacking in tensile strength. Such needles are unable to traverse thickened or tight tissue. The stiffer and stronger monopolar EMG needle electrode used in ETOIMS, with a diameter $0.41 \mathrm{~mm}$, allows treatment to deeper muscle layers.

Stretching of tight muscles and massage are common in rehabilitation to relieve pain, muscle tension and tenderness,

Abbreviations: ETOIMS, electrical twitch obtaining intramuscular stimulation; FABERE, flexion, abduction, and external rotation of the hip; MPS, myofascial pain syndrome; VAS, visual analogue scale 


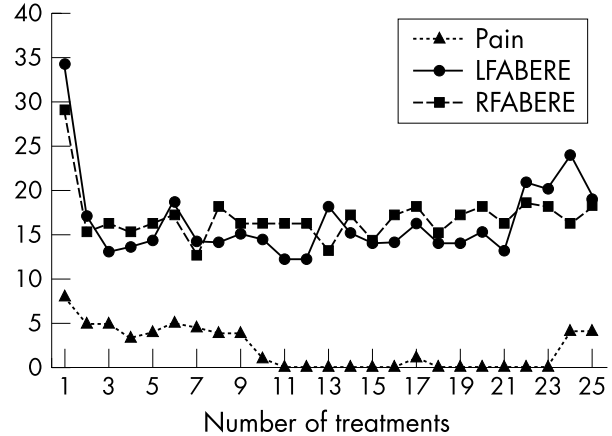

Figure 1 Relation between pain levels, (L/R)FABERE (ability to flex, abduct, and externally rotate the left or right hip), and the number of treatments.

and to enhance the range of motion. However, active physical training exercises are more useful than stretching in adductor muscle strains. ${ }^{7}$ Twitch contractions of ETOIMS deliver precise and effective neuromuscular training by brief active mobilisation and focused stretching of shortened deep muscle fibres at multiple treatment sites not readily accessible with standard treatments. Twitch muscle contractions with low frequency stimulation $(3 \mathrm{~Hz})$ also increase blood perfusion. ${ }^{8}$

Proprioceptive and sensorimotor stimulation of subconscious and automatic reflexes can provide activation of gluteal muscles to restore strength and gain pelvic stability in chronic lower back pain. ${ }^{9}$ Muscle contractions associated with ETOIMS can stimulate deeper motor end plate zones and potentially provide more effective activation of mechanoreceptors in joint ligaments.

A decreased range of motion may be caused by muscle shortening or pain, and muscle shortening predisposes to pain. FABERE assesses the flexibility of major muscles crossing the hip, knee, and lower back and is impaired in lower back pain. ${ }^{10}$ In spite of complete pain relief after 10 ETOIMS sessions, FABERE continues to be limited in our patient, indicating that chronic muscle tightness is more difficult to treat and may predispose the athlete to recurrent injuries. Although pain-free, the patient chose to continue long term ETOIMS treatment for symptoms of muscle tightness which helped to maintain range of motion, avoided pain recurrence, and provided satisfactory athletic performance.

\section{Take home message}

- Because of its ability to treat tender myofascial trigger points in many muscles, electrical twitch obtaining intramuscular stimulation (ETOIMS) has a place in treating sports related recurrent myofascial pain syndrome.

- In this case report, ETOIMS alleviated pain and improved athletic function in an elite football player whose symptoms failed to respond to standard treatments.

\section{Authors' affiliations}

J Chu, I Takehara, T-C Li, I Schwartz, Rehabilitation Medicine, Hospital of the University of Pennsylvania, Spruce Street, Philadelphia,

Pennsylvania, USA

Correspondence to: Dr J Chu, University of Pennsylvania Medical Center, Rehabilitation Medicine, Ground Floor White Building, 3400 Spruce Street, Philadelphia, PA 19104, USA; jechu@comcast.net

Accepted 9 December 2003

\section{REFERENCES}

1 Orchard J. Intrinsic and extrinsic risk factors for muscle strains in Australian football. Am J Sports Medicine 2000;28:297-300.

2 Harreby MRI, Neergard K, Hesselsoe G, et al. Are radiologic changes in the thoracic and lumbar spine of adolescents risk factors for low back pain in adults? A 25 -year prospective cohort study of 640 school children. Spine 1995; 20:2298-302

3 Chu J. Twitch-obtaining intramuscular stimulation: observations in the management of radiculopathic chronic low back pain. J Musculoskeletal Pain 1999;7:131-46.

4 Chu J, Neuhauser D, Schwartz I, et al. The efficacy of automated/electrical twitch obtaining intramuscular stimulation (ATOIMS/ETOIMS) for chronic pain control: evaluation with statistical process control methods. Electromyogr Clin Neurophysiol 2002;42:393-401.

5 Travell JG, Simons DG. Myofascial pain and dysfunction. The trigger point manual, vol 1. Upper half of body. Maryland: Williams and Wilkins, 1999.

6 Gunn CC. Treatment of chronic pain. Intramuscular stimulation for myofascial pain of radiculopathic origin. London: Churchill Livingstone, 1996.

7 Holmich P, Uhrskou P, Ulnits L, et al. Effectiveness of active physical training as treatment for long-standing adductor-related groin pain in athletes: randomised trial. Lancet 1999;353:439-43.

8 Janssen TW, Hopman MT. Blood flow response to electrically induced twitch and tetanic lower limb muscle contractions. Arch Phys Med Rehabil 2003;84:982-7.

9 Bullock-Saxton JE. Janda V. Bullock MI. Reflex activation of gluteal muscles in walking. An approach to restoration of muscle function for patients with lowback pain. Spine 1993;18:704-8.

10 Akkus S, Tamer MN, Yorgancigil H. A case of osteomalacia mimicking ankylosing spondylitis. Rheumatol Int 2001;20:239-42. 